\title{
Compte rendu de Objets d'art et art de l'objet en Océanie, de Dominique Barbe et al. (éds)
}

\section{Gilles Bounoure}

\section{OpenEdition}

1 Journals

\section{Édition électronique}

URL : http://journals.openedition.org/jso/7169

DOI : 10.4000/jso.7169

ISSN : 1760-7256

\section{Éditeur}

Société des océanistes

\section{Édition imprimée}

Date de publication : 15 décembre 2014

Pagination : $244-246$

ISBN : 978-2-85430-118-2

ISSN : 0300-953x

\section{Référence électronique}

Gilles Bounoure, «Compte rendu de Objets d'art et art de l'objet en Océanie, de Dominique Barbe et al. (éds) », Journal de la Société des Océanistes [En ligne], 138-139 | 2014, mis en ligne le 15 décembre 2014, consulté le 24 septembre 2020. URL : http://journals.openedition.org/jso/7169 ; DOI : https:// doi.org/10.4000/jso.7169

Ce document a été généré automatiquement le 24 septembre 2020.

(c) Tous droits réservés 


\title{
Compte rendu de Objets d'art et art de l'objet en Océanie, de Dominique Barbe et al. (éds)
}

\author{
Gilles Bounoure
}

\section{RÉFÉRENCE}

BARBE Dominique, Michel PÉREZ et René ZIMMER (éds), 2013. Objets d'art et art de l'objet en

Océanie, actes du xxI colloque Corail, Paris, L'Harmattan, 246 p., une vingtaine

d'illustrations noir et blanc.

1 Sous cet ambitieux intitulé et une couverture reproduisant à l'avers un détail de sculpture malangan dite totok attribuée au XIX ${ }^{e}$ siècle, issue d'une collection privée de Nouméa, et au dos une peinture du ni-Vanuatu Jérôme Sioom datée de 2008, ce volume rassemble quatorze contributions d'un colloque de la « Coordination pour l'Océanie des recherches sur les arts, les idées et les littératures » (Corail) réuni à Nouméa en juin 2009. Il est surprenant de lire, en tête et occupant près de $10 \%$ de l'ouvrage, deux essais n'ayant rien à voir, titre comme contenu, avec les arts océaniens (" Tour et retour des objets dans La Loi de Roger Vailland », par B.-F. Moschetto, et « Le rôle de la miniature de saint Paul de Thèbes dans Paul et Virginie » par M. Nováková). Il s'agissait peut-être d'illustrer les revers de l'exotisme, mais il serait aventureux d'avancer la moindre hypothèse sur leur présence dans ce livre, ni l'avant-propos de D. Barbe ni la préface de R. Zimmer ne présentant ces contributions ou les suivantes.

2 Avec « Peinture océanienne contemporaine et colonisation de l'imaginaire. L'exemple mélanésien » (pp. 41-53), D. Barbe avance une typologie distinguant «art premier » et " art premier contemporain », notion qu'il aurait été utile de définir; "l'art premier contemporain rejeté au rang de curios est par ailleurs handicapé par les politiques culturelles qui le figent au rang d'art au service de la nation ", d'où résulte « une double situation qui oblige les peintres à passer par les fourches caudines des galeries et du 
monde occidental». Dans "Ni antique ni moderne: contribution à une analyse des objets sur le marché de l'art » (pp.55-65), Sophie Cazaumayou prolonge des réflexions formulées dans son livre de 2007, Objets d'Océanie (voir jso $n^{\circ} 128$ ). À côté de points d'histoire mal vérifiés lui faisant affirmer par exemple (p. 64) que « dès 1914 des marchands comme Paul Guillaume ou Charles Ratton faisaient des publicités dans la presse coloniale pour l'achat de tels objets destinés à alimenter le marché parisien »Ratton n'en était alors qu'à commencer ses études d'histoire de l'art du Moyen Âge, interrompues par la Grande Guerre (Dagen, in Dagen et Murphy, 2013:13 et passim) et Guillaume à ouvrir sa première galerie - elle avance des propositions en vue d'appliquer aux objets océaniens circulant sur le marché « un vocabulaire dont la sémantique aura été définie avec rigueur. Dans le jargon des Beaux-Arts, "fait par", "école de", "suiveur de", "dans le goût de" situent avec rigueur le degré d'authenticité de l'œuvre par rapport à son identification présumée. Un effort de clarification dans cet esprit devient urgent » pour ce qui est des objets traditionnels d'Océanie.

Hélène Goiran, s'intéressant aux « armes traditionnelles fidjiennes : art de l'objet et art de la guerre» (pp.67-78), en présente une typologie illustrée s'appuyant essentiellement sur celle de Fergus Clunie (1977) mais la simplifiant beaucoup, mentionnant à peine arcs, lances et frondes. Avec «Chefs d'œuvre cachés en quête d'histoire» (pp. 99-108), Christian Coiffier résume trois recherches qu'il eut à mener sur des objets des collections du musée de l'Homme, une grande peinture mundugumor photographiée in situ par Margaret Mead et rapportée ensuite par l'équipage de La Korrigane, une sculpture anthropomorphe de Malakula photographiée in situ par John Layard et «apparue » en 1946 dans les collections du musée de l'Homme, et le célèbre "homme bleu» de Vao exposé au pavillon des Sessions. Aucune illustration n'accompagne malheureusement cette contribution. Dans «Tridacna gigas: objets de prestige en Mélanésie» (pp.109-122), l'antiquaire Didier Zanette reprend certaines pages du livre qu'il a consacré aux objets océaniens en bénitier et à leur confection, évoquant notamment ses "six ans de recherche et de démarches " pour obtenir du dernier initié les secrets de "la technique ancestrale du façonnage de la monnaie Boiken, rarement révélée jusqu'à ce jour ». Technique néanmoins décrite jadis par Finsch (1914: 79-92), plus précis que cet article, spécialement à propos des modes d'ébauchage, de perçage et de polissage (ibid. : 79-82) sur cette côte septentrionale de la Nouvelle-Guinée comme en d'autres aires de production que n'étudie pas D. Zanette.

La contribution de R. Zimmer, "Le casoar des contes et peintures papous" (pp.123-138), s'appuie pour l'essentiel sur une série de contes publiés par l'hebdomadaire œcuménique de Port Moresby Wantok et mettant en scène ce ratite devenu un emblème national de la Papouasie Nouvelle-Guinée. Ce début d'analyse pourrait être aisément prolongé, par exemple par l'examen des effets de l'orientation chrétienne de cette publication sur la matière même des contes, ou de ce qu'ils doivent eux-mêmes à une observation séculaire des mœurs de cet oiseau, justifiant le thème du "père nourricier» que relève R. Zimmer, mais sans les explications éthologiques qui s'imposaient. Texte ambitieux mais par trop dénué de références et d'exemples précis, "Hypersexuality in the Art of the Sepik» (pp.139-160) de M. Pérez s'inspire des formules de Richard L. Anderson plaçant les arts du Sepik sous le signe de «la puissance des esprits " combinée à "l'agression phallique », malheureusement sans discuter ce que pourrait être en art "l'hypersexualité », "l'hyposexualité " (les productions sulpiciennes?) ou la "moyenne sexualité » dont elles s'éloigneraient. Quant à qualifier ces arts de «masculins», de «misogynes» et finalement 
d'«androgynes », M. Pérez le fait après d'autres qu'il ne cite pas, et sans d'ailleurs définir ce qu'il englobe dans ces arts du Sepik où de bons spécialistes ont cru repérer des expressions artistiques moins « agressives » et «phalliques » que d'autres.

Dans «Le corps-objet, ou la mise en spectacle du corps australien»(pp.161-170), Chantal Kwost-Greff s'attache à l'exaltation par l'image du beach boy blanc australien au milieu du siècle dernier. Elle n'hésite pas à mettre en relation ces représentations passablement racistes avec les films de Leni Riefenstahl et l'idéologie qu'ils servaient, tout en exposant les circonstances qui rendirent ensuite cette imagerie obsolète, conduisant aussi à la faire oublier, utile rappel et intéressante plongée dans les archives méritant certainement d'être approfondis. «Le processus de création océanien: tradition-modernité, un faux débat?» (pp.171-202), de Nathalie Cartacheff, s'attache à la danse, à travers l'expérience de la chercheuse en Nouvelle-Calédonie. Le domaine est excellemment choisi, pour offrir avec le chant et plus largement les cérémonies les exemples les plus nets de cette interaction entre attachement à la tradition et appétit de la nouveauté qui assura la vitalité des arts anciens d'Océanie. On y lit d'utiles observations d'ethnographie "participative », sans pouvoir beaucoup adhérer à ce qui s'y extrapole pour l'ensemble des arts océaniens, faute d'éléments de comparaison suffisamment nombreux et consistants.

6 Avec «Définir l'art contemporain et ses enjeux culturels et politiques en Mélanésie » (pp. 171-223), Domitille Barbe étudie successivement « la mort de l'objet "authentique" et l'émergence de l'art contemporain», «l'esthétisation par l'exposition» et «la nouvelle authenticité de l'art contemporain» mélanésien, ambiguë puisqu'il reste surtout apprécié comme «tribal », « national » ou « indigène ». Florence Rortais, dans «De l'ethnologie aux arts plastiques: collectes, muséographies et inspirations contemporaines monographiques» (pp. 225-243), s'attache surtout aux « installations sensori-motrices et esthétiques » et autres «performances muséographiques » que la chercheuse a proposées depuis 2001 au musée de Nouvelle-Calédonie, posant «des questions définitivement ontologiques ouvrant le débat sur de nouveaux horizons réflexifs ", ainsi qu'elle écrit pour clore ce dernier exposé. On ne sait si telle est aussi l'opinion des éditeurs de ce volume, auquel ils n'ont pas donné d'autre conclusion.

7 De ce recueil disparate, accueillant de nombreux jeunes chercheurs dont les propositions appellent souvent examen et discussion - nul ne saurait s'en plaindre -, émerge, comme de qualité et d'intérêt très supérieurs, sinon comme exemple à proposer à d'autres participants de ce colloque, la contribution de Nicolas Garnier, «Le monde derrière des barreaux: sacs brodés de prisonniers, une iconographie carcérale en Papouasie Nouvelle-Guinée » (pp. 79-97), fruit d'un long travail de terrain dans les prisons de Port Moresby, Wewak, Chambri et Goroka. Elle se distingue par la précision des observations, la clarté de leur exposition, la sensibilité de l'ethnologue à décrire les prisonniers brodant leurs sacs de riz pour «tuer le temps » (kilim taim). On ne saurait résumer ici cet article (qu'on peut compléter par les remarques de Peter Solo Kinjap sur les bilum en fibres de plastique tressés par les femmes des Hautes Terres dans Thomas et Bolton 2013, pp. 104-110, ouvrage décrit dans le présent $J S O$ ), mais il justifie à lui seul la publication de cet ouvrage, dont il corrige aussi diverses formules trop hâtives. À suivre N. Garnier, il y a bien aujourd'hui en Océanie ce qu'on pourrait appeler un « art premier contemporain », n'étant pas « au service de la nation », ne procédant d'aucune « politique culturelle » et échappant pour l'instant aux « fourches caudines des galeries 
et du monde occidental », et l'on saisit ici ce que ces développements artistiques ont d'historique, dimension trop souvent négligée par les historiens de l'art.

\section{BIBLIOGRAPHIE}

CLUNIE Fergus, 1977. Fijian Weapons and Warfare, Suva, Bulletin of the Fiji Museum 2.

DAGEN Philippe et Maureen MURPHY (éds), 2013. Charles Ratton. L'invention des arts " primitifs », Paris, Skira-Flammarion-Musée du quai Branly.

FINSCH Otto, 1914. Südseearbeiten. Gewerbe- und Kunstfleiss, Tauschmittel und « Geld » der Eingeborenen..., Hamburg, L. Friederichsen \& Co. 